\title{
WEAK CHEBYSHEV SUBSPACES AND CONTINUOUS SELECTIONS FOR THE METRIC PROJECTION
}

\author{
BY
}

GÜNTHER NÜRNBERGER AND MANFRED SOMMER

\begin{abstract}
Let $G$ be an $n$-dimensional subspace of $C[a, b]$. It is shown that there exists a continuous selection for the metric projection if for each $f$ in $C[a, b]$ there exists exactly one alternation element $g_{f}$, i.e., a best approximation for $f$ such that for some $a<x_{0}<\cdots<x_{n}<b$,

$$
\varepsilon(-1)^{i}\left(f-g_{f}\right)\left(x_{i}\right)=\left\|f-g_{f}\right\|, \quad i=0, \ldots, n, \varepsilon= \pm 1 .
$$

Further it is shown that this condition is fulfilled if and only if $G$ is a weak Chebyshev subspace with the property that each $g$ in $G, g \neq 0$, has at most $n$ distinct zeros. These results generalize in a certain sense results of Lazar, Morris and Wulbert for $n=1$ and Brown for $n=5$.
\end{abstract}

If $G$ is a nonempty subset of a normed linear space $E$ then for each $f$ in $E$, we define $P_{G}(f):=\left\{g_{0} \in G:\left\|f-g_{0}\right\|=\inf \{\|f-g\|: g \in G\}\right\}$. $P_{G}$ defines a set-valued mapping of $E$ into $2^{G}$ which in the literature is called the metric projection onto $G$. A continuous mapping $s$ of $E$ into $G$ is called a continuous selection for the metric projection $P_{G}$ (or, more briefly, continuous selection) if $s(f)$ is in $P_{G}(f)$ for each $f$ in $E$. In this paper we treat the problem of the existence of continuous selections for $n$-dimensional subspaces $G$ of $C[a, b]$, with $C[a, b]$ as usual the Banach space of real-valued continuous functions on $[a, b]$ under the uniform norm.

A. Lazar, P. Morris and D. Wulbert [4] have characterized the 1-dimensional subspaces of $C(X)$ with $X$ compact Hausdorff, which admit a continuous selection. They raised the problem of characterizing the corresponding $n$-dimensional subspaces. The only known result for higher dimensional subspaces has been given by A. Brown [1], who has shown the existence of continuous selections for certain 5-dimensional subspaces of $C[-1,1]$.

To obtain continuous selections, Lazar, Morris and Wulbert [4] and Brown [1] proceeded as follows: For each $f$ in $C[a, b]$ they considered all $g$ in $P_{G}(f)$ which can be written as $g=a_{1} g_{1}+\cdots+a_{n} g_{n}$, where $g_{1}, \ldots, g_{n}$ is a basis

Received by the editors July 30, 1976.

AMS (MOS) subject classifications (1970). Primary 41A30, 46E15.

Key words and phrases. Continuous selection, metric projection, weak Chebyshev spaces, alternation elements. 
of $G$, and chose the unique element $g$ in $P_{G}(f)$ with maximal coefficient $a_{n}$. This works in the cases $n=1$ and $n=5$.

Using this kind of selection it does not seem possible to get a general theorem for $n$-dimensional subspaces in $C[a, b]$. With new methods, however, and in the setting of weak Chebyshev subspaces we can give a sufficient condition for the existence of continuous selections.

R. Jones and L. Karlovitz [2, Theorem 4] have shown that an ndimensional subspace $G$ of $C[a, b]$ is weak Chebyshev if and only if for each $f$ in $C[a, b]$ there exists at least one alternation element $g_{f}$ (see Definition 1 below) in $P_{G}(f)$. We show that if for each $f$ in $C[a, b]$ there exists exactly one alternation element $g_{f}$ in $P_{G}(f)$, then $s(f)=g_{f}$ defines a continuous selection (Proposition 2). From Theorem 8 and Theorem 11, which together represent the main result of this paper, it follows that for an $n$-dimensional weak Chebyshev subspace $G$ each $f$ in $C[a, b]$ has exactly one alternation element in $P_{G}(f)$ if and only if each $g \in G, g \neq 0$, has at most $n$ distinct zeroes. (In particular, $g$ may not vanish on intervals.)

Using this result and Proposition 2, we immediately get an existence theorem for continuous selections for $n$-dimensional subspaces (Corollary 9). Brown [1] uses essentially stronger conditions to guarantee the existence of continuous selections for 5-dimensional subspaces of $C[-1,1]$. Brown's result disproves a claim of Lazar, Morris and Wulbert [4], who tried to show that for $n$-dimensional subspaces $G$ in $C(X)(X$ a connected, compact, Hausdorff space) such that 1 is in $G$ and each $g$ in $G, g \neq 0$, does not vanish on an open set in $X$, there does not exist a nontrivial continuous selection.

Finally, Let us remark that from P. Schwartz [8] it follows that under the assumption of Corollary 9 the continuous selection is unique.

In the following let $G$ be an $n$-dimensional subspace of $C[a, b]$.

1. Definition. If $f$ is in $C[a, b]$, then $g$ in $P_{G}(f)$ is called an alternation element ( $A$-element) of $f$ if there exist $n+1$ distinct points $a \leqslant x_{0}<\cdots<$ $x_{n} \leqslant b$ such that

$$
\varepsilon(-1)^{i}(f-g)\left(x_{i}\right)=\|f-g\|, \quad i=0, \ldots, n, \varepsilon= \pm 1 .
$$

The points $a \leqslant x_{0}<\cdots<x_{n} \leqslant b$ are called alternating extreme points of $f-g$.

First, we want to show that when each $f$ has a unique $A$-element then we can always define a continuous selection.

2. Proposition. Suppose for each $f$ in $C[a, b]$ there exists exactly one $A$-element $g_{f}$ in $P_{G}(f)$. Define $s: C[a, b] \rightarrow G$ by $s(f)=g_{f}$ for each $f \in C[a, b]$. Then, $s$ is a continuous selection for $P_{G}: C[a, b] \rightarrow 2^{G}$.

Proof. We suppose $s$ is not continuous. 
Because of the finite dimensionality of $G$, there exist $f \in C[a, b], g \in G$ and a sequence $\left(f_{m}\right) \subset C[a, b]$ so that $f_{m} \rightarrow f, s\left(f_{m}\right) \rightarrow g$, but $g \neq s(f)$.

We will show that $g$ is an $A$-element of $f$ and this will contradict the uniqueness of the $A$-element.

By definition, $s\left(f_{m}\right)$ is an $A$-element of $f_{m}, m \in N$. Therefore, there are extreme points $a \leqslant x_{0}^{(m)}<x_{1}^{(m)}<\cdots<x_{n}^{(m)} \leqslant b$ of $f_{m}-s\left(f_{m}\right)$.

We can assume that

(1) $(-1)^{i}\left(f_{m}-s\left(f_{m}\right)\right)\left(x_{i}^{(m)}\right)=\left\|f_{m}-s\left(f_{m}\right)\right\|, \quad i=0, \ldots, n, m \in \mathbf{N}$.

Here it may be necessary to choose a subsequence of $\left(f_{m}\right)$ and perhaps work with $-f$ and $-f_{m}$ in place of $f$ and $f_{m}$. We can also assume (again choosing a subsequence if necessary) that $\lim _{m \rightarrow \infty} x_{i}^{(m)}=x_{i}$ exists, $i=0,1, \ldots, n$. Now, since $\lim _{m \rightarrow \infty} f_{m}=f$ and $\lim _{m \rightarrow \infty} s\left(f_{m}\right)=g$, we have

$$
\begin{aligned}
\|f-g\| & =\lim _{m \rightarrow \infty}\left\|f_{m}-s\left(f_{m}\right)\right\| \\
& =(-1)^{i} \lim _{m \rightarrow \infty}\left(f_{m}-s\left(f_{m}\right)\right)\left(x_{i}^{(m)}\right) \\
& =(-1)^{i}(f-g)\left(x_{i}\right)
\end{aligned}
$$

where in the second equality we used (1) and the uniform convergence. This shows that $g$ is an $A$-element, which is the desired contradiction.

Jones and Karlovitz [2] have characterized those $n$-dimensional subspaces of $C[a, b]$ which have at least one $A$-element for each $f$ in $C[a, b]$. For this characterization, we need the following definition:

3. Definition. $G$ is called weak Chebyshev if each $g$ in $G$ has at most $n-1$ changes of sign, i.e., there do not exist points $a<x_{0}<\cdots<x_{n} \leqslant b$ such that $g\left(x_{i}\right) \cdot g\left(x_{i+1}\right)<0, i=0, \ldots, n-1$.

Jones-Karlovitz [2] have proved the following theorem:

4. TheOREM. $G$ is weak Chebyshev if and only if for each $f$ in $C[a, b]$ there exists at least one $A$-element in $P_{G}(f)$.

To get a continuous selection under application of Proposition 2, we examine what additional conditions a weak Chebyshev subspace has to fulfill in order that each $f$ in $C[a, b]$ has exactly one $A$-element.

We need the following standard definition:

5. Definition. A zero $x_{0}$ of $f$ in $C[a, b]$ is said to be a simple zero if $f$ changes sign at $x_{0}$ or if $x_{0}=a$ or $x_{0}=b$.

A zero $x_{0}$ of $f$ in $C[a, b]$ is said to be a double zero if $f$ does not change sign at $x_{0}$ and $x_{0} \neq a, x_{0} \neq b$.

In the following, we count simple zeroes as one zero and double zeroes as two zeroes. To prove the following results we need the lemma below.

6. LEMMA. If $f$ is in $C[a, b]$ and if there exist $n+1$ points $a \leqslant x_{0}<\cdots<$ 
$x_{n} \leqslant b$ such that

$$
\varepsilon(-1)^{i} f\left(x_{i}\right) \geqslant 0, \quad i=0, \ldots, n, \varepsilon= \pm 1,
$$

then $f$ has at least $n$ zeroes $y_{i}$ such that

$$
x_{0} \leqslant y_{0} \leqslant x_{1} \leqslant y_{1} \leqslant \cdots \leqslant x_{n-1} \leqslant y_{n-1} \leqslant x_{n} .
$$

7. LEMMA. If $G$ is an $n$-dimensional weak Chebyshev subspace of $C[a, b]$ such that there exists a $g$ in $G, g \neq 0$, with at least $n+2$ zeroes, then there exists a $\tilde{g}$ in $G, \tilde{g} \neq 0$, with at least $n+1$ distinct zeroes.

Proof. Let $g$ be in $G, g \neq 0$, with at least $n+2$ zeroes in $[a, b]$, but only $r$, $r \leqslant n$, distinct zeroes. Suppose first that $g(a)=g(b)=0$, and set $\bar{x}:=$ $\max \{x \in[a, b) \mid g(x)=0\}$.

Let $a<x_{1}<\cdots<x_{s} \leqslant \bar{x}$ be the simple zeroes of $g$.

(a) $s+n-1$ is an even number.

We choose $n-1-s$ points $\bar{x}<x_{s+1}<\cdots<x_{n-1}<b$. Since $G$ is weak Chebyshev, by Jones and Karlovitz [2, p. 140] there exists a $\bar{g} \in G$, $\bar{g} \neq 0$, with

$$
\varepsilon(-1)^{i} \bar{g}(x) \geqslant 0, \quad x_{i-1}<x<x_{i}, \quad i=1, \ldots, n, \varepsilon= \pm 1,
$$

where $x_{0}=a, x_{n}=b$. By Lemma $6, \bar{g}$ has at least $n-1$ distinct zeroes. We choose $\varepsilon$ such that $\operatorname{sgn}(g(x) \cdot \bar{g}(x)) \geqslant 0$ if $x \in\left[a, x_{s+1}\right]$. Let $a=y_{1}<\cdots<$ $y_{t}=b$ be the distinct zeroes of $g$ in $[a, b]$.

Then

$$
M:=\min _{i=2, \ldots, t}\|g\|_{\left[y_{i-1} y_{i}\right]}>0 .
$$

We define $\tilde{g}:=M \bar{g} /(2\|\bar{g}\|)$.

The function $\tilde{g}$ has at least two further distinct zeroes in $[a, b]$, otherwise the function $g-\tilde{g}$ would have at least $n$ changes of sign. This would be a contradiction.

(b) $s+n-1$ is an odd number.

We choose $x_{0}=a$ and $n-s-2$ points

$$
\bar{x}<x_{s+1}<\cdots<x_{n-2}<b .
$$

Since $G$ has an $(n-1)$-dimensional weak Chebyshev subspace (see Sommer and Strauss [11, Theorem 2.6]), by Jones and Karlovitz [2, p. 140] there exists a $\bar{g} \in G, \bar{g} \neq 0$ with $\varepsilon(-1)^{i} \bar{g}(x) \geqslant 0, x_{i-1}<x<x_{i}, i=1, \ldots, n-1, \varepsilon=$ \pm 1 where $x_{n-1}=b$.

As before let $\operatorname{sgn}(g(x) \cdot \bar{g}(x)) \geqslant 0$ if $x \in\left[a, x_{s+1}\right]$.

Following (a) we construct a function $\tilde{g}$.

As before it follows that either the function $\tilde{g}$ or the function $g-\tilde{g}$ has $n+1$ distinct zeroes in $[a, b]$. 
If not $g(a)=g(b)=0$, the assertion can be shown in an analogous way. This completes the proof.

8. THEOREM. If $G$ is an n-dimensional weak Chebyshev subspace of $C[a, b]$ such that each $g$ in $G, g \neq 0$, has at most $n$ distinct zeroes, then each $f$ in $C[a, b]$ has exactly one A-element $g_{f}$ in $P_{G}(f)$.

Proof. Assumption. There exists a function $f$ in $C[a, b]$ which has two $A$-elements $g_{1}$ and $g_{2}$ in $P_{G}(f)$.

Let $a \leqslant x_{0}<\cdots<x_{n} \leqslant b$ be $n+1$ alternating extreme points of $f-g_{1}$ and let $a \leqslant y_{0}<\cdots<y_{n} \leqslant b$ be $n+1$ alternating extreme points of $f-g_{2}$.

We distinguish two cases:

First case.

$$
\begin{array}{ll}
(-1)^{i}\left(f-g_{1}\right)\left(x_{i}\right)=\left\|f-g_{1}\right\|, & i=0, \ldots, n, \\
(-1)^{i}\left(f-g_{2}\right)\left(y_{i}\right)=\left\|f-g_{2}\right\|, & i=0, \ldots, n .
\end{array}
$$

Then

$$
\begin{array}{ll}
(-1)^{i}\left(g_{2}-g_{1}\right)\left(x_{i}\right) \geqslant 0, & i=0, \ldots, n, \\
(-1)^{i}\left(g_{2}-g_{1}\right)\left(y_{i}\right) \leqslant 0, & i=0, \ldots, n .
\end{array}
$$

We treat only the case

$$
x_{i-2} \leqslant y_{i} \leqslant x_{i+2}, \quad i=0, \ldots, n,
$$

where the points $x_{i}$ for $i=-2,-1, n+1, n+2$ are omitted. In the other case, if $y_{i}<x_{i-2}$ for some $i$, we choose the points $y_{0}, \ldots, y_{i}, x_{i-2}, \ldots, x_{n}$ fulfilling

$$
\begin{aligned}
(-1)^{j}\left(g_{2}-g_{1}\right)\left(y_{j}\right) \leqslant 0, & j=0, \ldots, i, \\
(-1)^{j}\left(g_{2}-g_{1}\right)\left(x_{j-3}\right) \leqslant 0, & j=i+1, \ldots, n+3 .
\end{aligned}
$$

By Lemma $6, g_{2}-g_{1}$ has at least $n+3$ zeroes. Applying Lemma 7 we get a contradiction of the hypothesis that elements of $G$ have at most $n$ distinct zeroes.

A similar argument works for $x_{i+2}<y_{i}$.

Now we prove by induction that $g_{1}-g_{2}$ has at least $n+1$ distinct zeroes. This is a contradiction of the hypothesis on $G$. If $x_{i}=y_{i}, i=0, \ldots, n$, then $\left(g_{1}-g_{2}\right)\left(x_{i}\right)=0, i=0, \ldots, n$. We may assume $x_{i}<y_{i}$ for some $i=$ $\{0, \ldots, n\}$.

We show: $x_{j} \leqslant y_{j}, j=0, \ldots, n$.

If $y_{j_{0}}<x_{j_{0}}$ for any $j_{0} \in\{0, \ldots, n\}$ we choose

$$
y_{0}, \ldots, y_{j_{0}}, x_{j_{0}}, \ldots, x_{i}, y_{i}, \ldots, y_{n} \text { if } j_{0}<i
$$

and 


$$
x_{0}, \ldots, x_{i}, y_{i}, \ldots, y_{j_{0}}, x_{j_{0}}, \ldots, x_{n} \text { if } j_{0}>i \text {. }
$$

Because of (i) in both cases $g_{1}-g_{2}$ has at least $n+2$ zeroes by Lemma 6 . Applying Lemma 7 we get a contradiction of the hypothesis on $G$.

Now we show by induction that $g_{1}-g_{2}$ has at least $n+1$ distinct zeroes in $\left[x_{0}, y_{n}\right]: n=1$.

If $x_{0} \leqslant y_{0}<x_{1} \leqslant y_{1}$ (respectively $x_{0}<y_{0}=x_{1}<y_{1}$ or $x_{0}<x_{1}<y_{0}<$ $y_{1}$ ), then $g_{1}-g_{2}$ has one zero in each interval $\left[x_{0}, y_{0}\right],\left[x_{1}, y_{1}\right]$ (respectively $\left[x_{0}, y_{0}\right),\left(x_{1}, y_{1}\right]$ or $\left.\left[x_{0}, x_{1}\right],\left[y_{0}, y_{1}\right]\right)$.

Let the statement be true for $n-1$.

If $y_{n-1}<x_{n} \leqslant y_{n}$, then by assumption $g_{1}-g_{2}$ has $n$ distinct zeroes in $\left[x_{0}, y_{n-1}\right]$ and a further zero in $\left[x_{n}, y_{n}\right]$.

If $y_{n-1}=x_{n}<y_{n}$, then by assumption $g_{1}-g_{2}$ has $n$ distinct zeroes in $\left[x_{0}, y_{n-1}\right)$ and a further zero in $\left(x_{n}, y_{n}\right]$.

Finally we consider the case $x_{n}<y_{n-1}<y_{n}$ :

Since $(-1)^{n}\left(g_{2}-g_{1}\right)\left(x_{n}\right) \geqslant 0,(-1)^{n}\left(g_{2}-g_{1}\right)\left(y_{n-1}\right) \geqslant 0$, and $y_{n-2} \leqslant x_{n}$ we conclude as in the case $y_{n-1}<x_{n} \leqslant y_{n}$.

Second case.

$$
\begin{aligned}
(-1)^{i}\left(f-g_{1}\right)\left(x_{i}\right) & =\left\|f-g_{1}\right\|, & i=0, \ldots, n, \\
-(-1)^{i}\left(f-g_{2}\right)\left(y_{i}\right) & =\left\|f-g_{2}\right\|, & i=0, \ldots, n .
\end{aligned}
$$

We treat only the case that $f-g_{1}$ and

(iii) $\quad f-g_{2}$ have exactly $n+1$ alternating extreme points.

Otherwise we can apply the first case.

Then

$$
\begin{array}{ll}
(-1)^{i}\left(g_{2}-g_{1}\right)\left(x_{i}\right) \geqslant 0, & i=0, \ldots, n, \\
(-1)^{i}\left(g_{2}-g_{1}\right)\left(y_{i}\right) \geqslant 0, & i=0, \ldots, n .
\end{array}
$$

It is now enough to treat the case

$$
x_{i-1} \leqslant y_{i} \leqslant x_{i+1}, \quad i=0, \ldots, n,
$$

where the points $x_{-1}$ and $x_{n+1}$ are omitted. Otherwise we can conclude as in the first case. Applying the first case to the points $x_{0}, \ldots, x_{n-1}, y_{1}, \ldots, y_{n}$ because of (v) $g_{1}-g_{2}$ has $n$ distinct zeroes $z_{1}, \ldots, z_{n}$ in $\left[x_{0}, y_{n}\right]$.

We first prove: $z_{1}, \ldots, z_{n} \in(a, b)$. If $z_{1}=x_{0}$, then $y_{0}<x_{0}$. Otherwise $f-g_{2}$ has $n+2$ alternating extreme points $x_{0}, y_{0}, \ldots, y_{n}$. This is a contradiction to (iii). Therefore $z_{1}>a$.

If $z_{n}=y_{n}$, then $x_{n}>y_{n}$. Otherwise $f-g_{1}$ has $n+2$ alternating extreme points $x_{0}, \ldots, x_{n}, y_{n}$. This is a contradiction to (iii). Therefore $z_{n}<b$.

If $g_{1}-g_{2}$ has $n+1$ distinct zeroes, then we would get a contradiction of the hypothesis on $G$. 
Therefore we know $g_{1}-g_{2}$ has no further zero in $[a, b]$. Because of $a<z_{1}<\cdots<z_{n}<b$ and $G$ weak Chebyshev $g_{1}-g_{2}$ has at most $n-1$ changes of sign under the points $z_{i}$. We show $g_{1}-g_{2}$ has at most $n-2$ changes of sign:

If $g_{1}-g_{2}$ has $n-1$ changes of sign under the points $z_{i}$, then there exists exactly one zero $z_{j} \in(a, b)$ such that $g_{1}-g_{2}$ does not change sign at $z_{j}$.

Then it holds: If $z_{1}>x_{0}$, then because of (iv)

$$
\begin{array}{ll}
(-1)^{0}\left(g_{2}-g_{1}\right)(x)>0 & \text { if } a<x<z_{1} \text { and finally } \\
(-1)^{n}\left(g_{2}-g_{1}\right)(x)<0 & \text { if } z_{n}<x<b .
\end{array}
$$

If $z_{1}=x_{0}$, then $y_{0}<x_{0}$ and (vi) is also valid.

Now we get a contradiction to (iv):

$$
x_{n} \geqslant y_{n} \text {. }
$$

Then $z_{n}<x_{n}$ and because of (iv) $(-1)^{n}\left(g_{2}-g_{1}\right)\left(x_{n}\right)>0$. This is a contradiction.

$$
x_{n}<y_{n} .
$$

If $z_{n}<y_{n}$ we also get a contradiction because of (iv). But if $z_{n}=y_{n}$, then $x_{n}>y_{n}$ is always valid because of (iii).

We have shown:

If $g_{1}-g_{2}$ has exactly $n$ distinct zeroes, then $g_{1}-g_{2}$ has at most $n-2$ changes of sign. But in this case there exist $n+2$ zeroes of $g_{1}-g_{2}$ because of $a<z_{1}, z_{n}<b$.

Applying Lemma 7 we get a contradiction to the assumption.

Schwartz [8] has shown that for an $n$-dimensional subspace $G$ of $C(X)$ with the property that no $g$ in $G, g \neq 0$, vanishes identically on an open subset of $X$, the set of functions in $C(X)$ having unique best approximation in $G$ is dense in $C(X)$. Therefore there exists at most one continuous selection. By this result, Proposition 2 and Theorem 8 the next corollary follows immediately.

9. COROLlary. If $G$ is an n-dimensional weak Chebyshev subspace such that each $g$ in $G, g \neq 0$, has at most $n$ distinct zeroes, then there exists a unique continuous selection s: $C[a, b] \rightarrow G$ for $P_{G}: C[a, b] \rightarrow 2^{G}$.

Now we will give some nontrivial examples of subspaces $G$ in $C[a, b]$ fulfilling the assumption of Corollary 9.

10. Examples. (a) $G:=\left\langle x, x^{2}, \ldots, x^{n}\right\rangle \subset C[0,1]$. $G$ is Chebyshev in $(0,1]$ and therefore the assumption of Corollary 9 is fulfilled, but $G$ is not Chebyshev in $[0,1]$.

(b) For $n \geqslant 2$ and $n$ even, we define $G$ : $=\left\langle 1, x\left(1-x^{2}\right), x^{2}, x^{3}\left(1-x^{2}\right)\right.$, $\left.x^{4}, \ldots, x^{n-1}\left(1-x^{2}\right), x^{n}\right\rangle \subset C[-1,1]$. The dimension of $G$ is $n+1$. Each 
function $g$ in $G$ is a polynomial of degree $\leqslant n+1$ and has therefore at most $n+1$ zeroes in $[-1,1]$. Such a function $g$ can be written as $g=g_{1}-g_{2}$ where

$$
g_{1}(x)=\sum_{i=0}^{n / 2} a_{2 i} x^{2 i} \text { and } g_{2}(x)=x\left(1-x^{2}\right) \sum_{i=1}^{n / 2} a_{2 i-1} x^{2 i-2} .
$$

Because of the behaviour of $g_{1}(x)$ and $g_{2}(x)$ for $x \rightarrow \pm \infty$ it can be shown that $g_{1}-g_{2}$ has a zero in $(-\infty,-1] \cup(1, \infty)$. Therefore $G$ is Chebyshev in $(-1,1]$.

$G$ is not Chebyshev in $[-1,1]$ because there exists a function

$$
g_{0}(x)=x\left(1-x^{2}\right) \sum_{i=1}^{n / 2} a_{2 i-1} x^{2 i-2} \text { in } G, g \neq 0,
$$

having exactly $n+1$ zeroes in $[-1,1]$.

A similar example has been given by Brown [1] in the case $n=5$.

(c) $G:=\left\langle|x|, x^{3}\right\rangle \subset C[-1,1] . G$ is weak Chebyshev and each $g \in G$, $g \neq 0$, has at most 2 distinct zeroes, but $G$ is not Chebyshev in $[-1,1)$ or $(-1,1]$.

Finally we ask how strong the assumption of Theorem 7 is for the uniqueness of $A$-elements and we show that this is the weakest condition because the converse of Theorem 7 is true.

11. THEOREM. If $G$ is an n-dimensional weak Chebyshev subspace of $C[a, b]$ such that for each $f$ in $C[a, b]$ there exists exactly one $A$-element in $P_{G}(f)$ then each $g$ in $G, g \neq 0$, has at most $n$ distinct zeroes.

Proof. Assumption. There exists a $\tilde{g}_{0}$ in $G, \tilde{g}_{0} \not \equiv 0$, with at least $n+1$ distinct zeroes.

We define: $g_{0}:=\tilde{g}_{0} /\left\|\tilde{g}_{0}\right\|$. Then $\left\|g_{0}\right\|=1$.

Since $G$ is weak Chebyshev, $g_{0}$ has at most $n-1$ changes of sign. Therefore $n+1$ distinct zeroes $x_{0}, \ldots, x_{n}$ of $g_{0}$ exist such that $\varepsilon_{i} g_{0}(x) \geqslant 0$, $x \in\left[x_{i}, x_{i+1}\right], i=-1,0, \ldots, n, \varepsilon_{i}= \pm 1, x_{-1}:=a, x_{n+1}:=b$.

We construct a function $f$ in $C[a, b]$, having two $A$-elements in $P_{G}(f)$. We define $f$ in the following way:

$$
\begin{gathered}
\varepsilon_{-1}(-1)^{i} f\left(x_{i}\right)=1, \quad i=0, \ldots, n, \\
\|f\|=1, \\
0, g_{0} \text { in } P_{G}(f) .
\end{gathered}
$$

Then $g_{0}$ and 0 are $A$-elements of $f$.

Construction of $f$ :

(a) We may assume $g \geqslant 0$ for $x \in\left[a, x_{0}\right]$.

We define: $f(x):=1$ if $x \in\left[a, x_{0}\right],(-1)^{i} f\left(x_{i}\right)=1, i=0, \ldots, n$. 
(b) Definition of $f$ in $\left[x_{0}, x_{1}\right]$ :

First case. $g_{0}(x) \geqslant 0$ if $x \in\left[x_{0}, x_{1}\right]$.

Let $\tilde{x}:=\left(x_{0}+x_{1}\right) / 2$ and $f(\tilde{x}):=0$.

Let $f$ be linear in $\left[x_{0}, \tilde{x}\right]$

$$
f(x):=g_{0}(x)-g_{0}(\tilde{x})+2\left(g_{0}(\tilde{x})-1\right) \frac{x-\tilde{x}}{x_{1}-x_{0}} \quad \text { if } x \in\left[\tilde{x}, x_{1}\right] .
$$

Second case. $g_{0}(x) \leqslant 0$ for $x \in\left[x_{0}, x_{1}\right]$.

$$
\begin{gathered}
f(x):=g_{0}(x)-g_{0}(\tilde{x})+\left(1+g_{0}(\tilde{x})\right) \frac{\tilde{x}-x}{\tilde{x}-x_{0}} \quad \text { if } x \in\left[x_{0}, \tilde{x}\right], \\
f(\tilde{x}):=0 .
\end{gathered}
$$

Let $f$ be linear in $\left[\tilde{x}, x_{1}\right]$.

This construction of $f$ is continued in an analogous way for the intervals $\left[x_{1}, x_{2}\right], \ldots,\left[x_{n-1}, x_{n}\right],\left[x_{n}, b\right]$. Obviously $f$ is continuous in $[a, b]$.

We show: $|f(x)| \leqslant 1$ if $x \in\left[x_{0}, x_{1}\right]$.

In the first case:

$$
\begin{aligned}
-1 & \leqslant g_{0}(x)-g_{0}(\tilde{x})+g_{0}(\tilde{x})-1 \\
& \leqslant g_{0}(x)-g_{0}(\tilde{x})+2\left(g_{0}(\tilde{x})-1\right) \frac{x-\tilde{x}}{x_{1}-x_{0}} \\
& =f(x) \leqslant g_{0}(x)-g_{0}(\tilde{x}) \leqslant g_{0}(x) \leqslant 1 \text { if } \tilde{x} \in\left[x, x_{1}\right] .
\end{aligned}
$$

In the second case:

$$
\begin{aligned}
-1 & \leqslant g_{0}(x) \leqslant g_{0}(x)-g_{0}(\tilde{x}) \\
& \leqslant g_{0}(x)-g_{0}(\tilde{x})+\left(1+g_{0}(\tilde{x})\right) \frac{\tilde{x}-x}{\tilde{x}-x_{0}} \\
& =f(x) \leqslant g_{0}(x)-g_{0}(\tilde{x})+\left(1+g_{0}(\tilde{x})\right) \\
& \leqslant g_{0}(x)+1 \leqslant 1 \quad \text { if } x \in\left[x_{0}, \tilde{x}\right] .
\end{aligned}
$$

Therefore $|f(x)| \leqslant 1$ if $x \in\left[x_{0}, x_{1}\right]$.

We can show in an analogous way: $\left|f(x)-g_{0}(x)\right| \leqslant 1$ if $x \in\left[x_{0}, x_{1}\right]$. These estimations hold in each interval because of the construction of $f$.

Therefore $f-0$ and $f-g_{0}$ have $x_{0}, \ldots, x_{n}$ as alternating extreme points.

If 0 and $g_{0}$ are not in $P_{G}(f)$, then there would exist a function $g$ in $G$ such that $\|f-g\|<\|f\|=\left\|f-g_{0}\right\|=1$. Since $(-1)^{i} f\left(x_{i}\right)=1=\|f\|>$ $(-1)^{i}\left(f\left(x_{i}\right)-g\left(x_{i}\right)\right)$ it follows $(-1)^{i} g\left(x_{i}\right)>0, i=0, \ldots, n$.

Hence $g$ has at least $n$ changes of sign in $[a, b]$. This is a contradiction to the assumption that $G$ is weak Chebyshev. Therefore 0 and $g_{0}$ are two $A$-elements of $f$ in $P_{G}(f)$.

This completes the proof.

Finally we show in Proposition 14 that a large class of weak Chebyshev 
subspaces in $C[a, b]$ whose nonzero functions have only finitely many zeroes fulfill the assumption of Corollary 9 and therefore admit a unique continuous selection.

We need the following definition (cf. Singer [10, p. 126]):

12. Definition. A linear subspace $G$ of a normed linear space $E$ is called $k$-Chebyshev (where $k$ is an integer with $0 \leqslant k \leqslant \infty$ ), if for each $f$ in $E$ we have $0 \leqslant \operatorname{dim} P_{G}(f) \leqslant k$.

Finite-dimensional $k$-Chebyshev subspaces in $C(X)$ ( $X$ compact) are characterized in Singer [10, p. 240]:

13. THEOREM. If $G$ is an n-dimensional subspace of $C(X)(X$ compact) and $k$ an integer with $0 \leqslant k \leqslant n-1$. Then $G$ is a $k$-Chebyshev subspace if and only if there do not exist $n-k$ distinct points $x_{1}, \ldots, x_{n-k}$ in $X$ and $k+1$ linearly independent functions $g_{0}, g_{1}, \ldots, g_{k}$ in $G$, such that

$$
g_{i}\left(x_{j}\right)=0, \quad j=1, \ldots, n-k, i=0,1, \ldots, k .
$$

Using the methods in the proof of Lemma 7 and Theorem 13 we can show in a straightforward manner that the following Proposition holds:

14. Proposition. If $G$ is an $n$-dimensional, weak Chebyshev subspace which is $(n-1)$-Chebyshev and if each $g$ in $G, g \neq 0$, has only finitely many zeroes, then each $g$ in $G, g \neq 0$, has at most $n$ distinct zeroes.

\section{REFERENCES}

1. A. L. Brown, On continuous selections for metric projections in spaces of continuous functions, J. Functional Analysis 8 (1971), 431-449. MR 45 \#5725.

2. R. C. Jones and L. A. Karlovitz, Equioscillation under nonuniqueness in the approximation of continuous functions, J. Approximation Theory 3 (1970), 138-145. MR 41 \#8899.

3. S. Karlin and W. J. Studden, Tchebycheff systems: With applications in analysis and statistics, Interscience, New York, 1966. MR 34 \#4757.

4. A. J. Lazar, D. E. Wulbert and P. D. Morris, Continuous selections for metric projections, J. Functional Analysis 3 (1969), 193-216. MR 39 \#3288.

5. G. Meinardus, Approximation of functions: Theory and numerical methods, Springer-Verlag, New York, 1967. MR 36 \# 571.

6. G. Nürnberger, Dualität von Schnitten für die metrische Projektion und von Fortsetzungen kompakter Operatoren, Dissertation, Erlangen, 1975.

7.

8. P. Schwartz, Almost-Chebyshev subspaces of finite dimension in $C(Q)$, (preprint).

9. I. Singer, Best approximation in normed linear spaces by elements of linear subspaces, Springer-Verlag, Berlin and New York, 1970. MR 42 \# 4937.

10. The theory of best approximation and functional analysis, CBMS Regional Conf. Ser. in Appl. Math., vol. 13, SIAM, Philadelphia, 1974. MR 51 \# 10967.

11. M. Sommer and H. Strauss, Eigenschaften von schwach tschebyscheffschen Räumen, J. Approximation Theory (to appear).

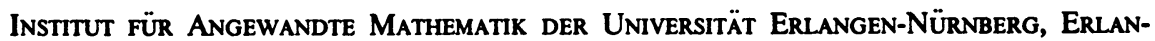
gen, Federal Republic of Germany 